Mater. Sci. Bull., Vol, 4, No. 3, May 1982, pp. 297-316. (C) Printed in India.

\title{
The onset of transient Marangoni convection in a liquid layer subjected to rotation about a vertical axis
}

\author{
N RUDRAIAH \\ UGC-DSA centre in Fluid Mechanics, Department of Mathematics, Central College \\ Bangalore University, Bangalore 560001 , India \\ MS reccived 17 Junc 1980
}

\begin{abstract}
Alsstract. In vicw of the interesting possibilitics of controlling surface tension-driven convection, anticipated in space cxperiments involving fluid interfaces, the problem of the stability of a thin horizontal fluid layer subjected to rotation about a vertical axis, when the thermal (or concentration) gradient is not uniform is examincd by linear stability analysis. Attention is focussed on the situation where the critical Marangoni number is greater than that for the case of uniform thermal gradient and the convection is not, in general, maintained. The case of adiabatic boundary condition is examined becausc it brings out the effect of surface tension at the free surfaces and allows a simple application of the Galerkin technique, which gives useful results. Numerical results are obtained for special cases and some general conclusions about the destabilizing effects of various basic temperature profiles and the stabilizing effect of corialis force are presented. The results indicate that the most destabilizing temperature gradient is one for which the temperature gradient is a step function of the depth. Increase in Taylor number and the inverted parabolic basic temperature profile suppress the onset of convection.
\end{abstract}

Keywords. Marangoni convection, rotation.

\section{Introduction}

The determination of the criterion for the onset of convection under microgravity condition, i.c., gravity reduced by several orders of magnitude ranging frcm $10^{-3} \mathrm{~g}$ to $10^{-6} \mathrm{~g}$, has considerable interest in material science pracessing in space. It is usually believed (see Ostrach 1979; Polezhaev 1979) that natural convection cannot occur in a microgravity environment and heat and mass transfer will be exercised by molecular conductivity and diffusion. This is not always true (Ostrach 1979). Convection driven by surface tension gradients is inevitable in material science experimental configurations in space missions, because such configurations often involve fluid interfaces. Experiments on the Apollo 14 and 17 flights (Grodzka and Bannister 1972; Bannister et al 1973) have shown that convection can still be induced by surface tension effects, even if buoyancy forces are absent. Neglecting such a convection may cause considerable crrors in setting up experjments in space and interpreting their results. Hence, for material science processing in space, it is important to evaluate the critical Marangoni number below which canvection cannot accur and to suggest the mechanisms to suppress convection.

M.S. -8 
In small scale fluid mechanics, the fact that interfacial regions between fluid phases play an important part in driving as well as impeding convection, was abserved for the first time by Block (1956). Pearson (1958) gave a detailed mathematical analysis for the onset of convection driven by surface tension gradients. Later, Sternling and Scriven (1959) and Scriven and Sternling $(1960,1964)$ examined the onset of steady cellular convection driven by surface tension gradients as an extension of Pearson's (1958) stability analysis. Nield (1975) examined the onset of transient convective instability driven by surface tension using the Galerkin methad. But the effect of coriolis force on the surface tension driven convection has not been given much attention. Recently, Sarma (1979) has investigated the problem of thermacapillary stability of a thin liquid layer keated uniformly from below subjected to Iotation about the transverse axis. He has illustrated the vital role of the different boundary conditions and the destabilising character of the long-wave disturbances at the fluid-fluid interface using a neutral stability curve based on analytical solutions of the pertinent eigenvalue problem. These results pertain to basic uniform temperature gradient. In a weightlessness environment, however, it is difficult to maintain a basic uniform temperature gradient. There is usually sudden heating or cooling giving rise to a non-uniform basic temperature gradient. The effect of non-uniform temperature gradient on surface tension driven convection with ratation about the vertical axis has not been given much attention. This is investigated in this paper using the single term Galerkin expansion and considering adiabatic temperature conditions at the boundaries.

Before investigating this problem, let us explain briefly the different mechanisms of generating convection. For practical applications, convective processes are suitably divided into two categories: (a) buoyancy-induced processes-which depend directly on the gravity $\vec{g}$ and (b) non-gravitational processes-which depend an surface tension gradients and which are relevant to microgravity conditions.

The basic principles underlying these processes are outlined below.

\subsection{Convection induced by buoyancy}

Usually there are two types of convection, viz., forced convections and natural or free convection. Here, we consider only free convection induced by the density gradient. The density gradient may be either parallel to the gravity but opposing it or narmal to it. The former is usually called Rayleigh-Benard convection and the latter is called conventional convection or Ober-Beck convection (Joseph 1976) where the mation is spontaneous. We explain here only the physical mechanism of Rayleigh-Benard conveotion.

Consider a fluid layer occupying the space between two parallel planes, infinite in horizontal extent separated by a distance $d$ apart, heated uniformly from below and cooled from above $\left(T_{2}>T_{1}\right)$. This means that the density of the top layer is higher than that of the bottom layer and we expect that the mation starts immediately as in conventional convection and converts the internal energy to kinetic energy. This is not so because, initially the temperature difference is not sufficient to overcome the viscous and thermal dissipations. But with increasing temperature difterence, a marginal condition is reached at some point 
in the layer at which it overcomes the viscous and thermal dissipations and the motion starts. The physical mechanism is that the cold fluid above moves downwards and the hot fluid below moves upwards resulting in the release of potential energy which can provide kinetic energy for the mation. Thus, there is a possibility that the equilibrium is unstable. Flow thus arises not because of the absence of equilibrium but because the equilibrium is unstable. A fluid heated from below is less dense at the bottom and therefore unstable. However, the viscosity, thermal conductivity and boundaries act to stabilise the fluid and create a threshold thermal gradient above which convection occurs and below which the fluid is in a quiescent state. The point at which the quiescent state breaks down and the motion starts is called the critical point and the corresponding temperature gradient is called the critical temperature gradient. Below this critical temperature gradient the fluid remains at rest and above it comes into motion. The fluid establishes hot rising regions and cold falling regions with horizontal motion at the top and bottom to maintain continuity. If there is no external constraint on the system to offset the destabilising nature of potential energy, the pattern of convection is uniform, i.e., the cell patterns will not be distorted and we say that the principle of exchange of stability is valid. This means that the velocity and temperature fields are in phase so that the rising fluid loses its heat by thermal conduction when it gets near to the cold top wall and can thus move downwards again. Similarly, the cold down-going fluid is warmed near the hot bottom wall and can rise again. So, when the flow is established as a steady pattern the continuous release of potential energy is balanced by viscous dissipation of mechanical energy. The potential energy is provided by the heating from below and cooling from above. This is usually called the marginal state. If there is an additional constraint like rotation, magnetic field or salinity on the system, the velocity and temperature fields will be out of phase and a part of the potential energy is now balanced by the constraint on the system and hence in the marginal state, the effect of additional constraint is to inhibit the onset of convection. However, in the time-dependent motion, a part of the constraint is now balanced by the local acceleration and less constrained effect is available to balance the potential energy and oscillatory or overstable convection sets in at a lower Rayleigh number than that of the marginal state. Overstable motion will also be responsible for finite amplitude motion. Therefore, in the study of onset of convection, ane has to consider (i) the marginal state, (ii) the overstable state, (iii) finite and large amplitude steady or overstable motions.

The systematic study of these involves the following three aspects:

(i) The determination of the condition for the onset of convection, which depends on the magnitude of the temperature difference. This is expressed in dimensionless form as the critical value of the Rayleigh number. The prablem here is to find the critical Rayleigh number at which the quiescent state breaks down. This is usually the realm of linear theory based on an infinitesimal perturbation and is explained in detail by Chandrasekhar (1961) for the case of a uniform temperature gradient. The critical Rayleigh number in the case of nonlinear theory based on arbitrary perturbations, usually called universal stability, is determined using the Liapunov direct method (Joseph 1976; Rudraiah 1972; Rudraiah and Prabhamani 1973, 1974a, b). 
(ii) The nature of cell pattern

The physically feasible cell pattern is the one which transfers maximum heat. In the quiescent state the transfer of heat is purely by canduction in the absence of radiation. If $H$ is the rate of heat transfer per unit arca, then $H=k \Delta T / d$, where $K$ is the thermal conductivity, $\triangle T$ is the temperature difference and $d$ is the distance between the plates. When the quiescent state breaks down, the heat transfer is both by conduction and convection and is expressed by the dimensionless parameter

$$
\mathrm{Nu}=H_{1} / H
$$

called the Nusselt number and $H_{t}$ is the sum of the heat transfer by conduction and convection. The variation of $\mathrm{Nu}$ with the Rayleigh number, $\mathrm{Ra}$, is called the heat transport curve. Nu remains equal to unity upto the critical Rayleigh number but increases above unity with the onset of convection. In practice $\mathrm{Nu}$ is determined for two and three-dimensional plan-forms. Of these, the one which gives the highest value of $\mathrm{Nu}$ is called the physically feasible cell pattern. The detailed structure of the plan-form is mathematically complicated because of the non-linear nature of the problem. A discussion of the question of the preferred cell shape, however, has been given by Palm (1960) taking into account the effects of non-linearity and the variation of viscasity. This is further developed by Segel and Stuart (1962) and Stuart (1964). The value of $\mathrm{Nu}$ is determined by calculating the amplitude of motion. The linear theory cannot predict the amplitude and we have to resart to the nonlinear theory. The theory of stability of nonlinear motion based on arbitrary perturbations is called universal stability (Joseph 1976). This universal stability predicts only the critical Rayleigh number and not the amplitude. Therefore, to determine the amplitude of motion, one has to resart to the local nonlinear stability analysis which is pivated on the linear theory. This lacal nonlinear stability is usually investigated using the Galerkin technique (Finalyson 1972; Rudraiah and Vortmeyer 1978), Fourier's truncated representation (Veronis 1966) and the power integral technique (Stuart 1958). The power integral technique was first proposed by Stuart (1958) and later it was applied to convection problems by many authors (Malkus and Veronis 1958: Rudraiah and Srimani 1980).

\section{(iii) Relative stability criterion}

We know that convection sets in at the critical Rayleigh number; below which there exists a unique basic solution, but above it, in addition to this, many possible solutions are possible depending on various possible wave number's and cell shapes. The question is, of the many solutions above the critical value, which is the stable one ? To answer this question, a relative stability criterion, has to be investigated (Malkus and Veronis 1958; Rudraiah and Srimani 1980). This is based an the result that when all the solutions are orthogonal to each other the fluid chooses that solution which has the maximum value of heat transport as the stable one. Although a detailed understanding of the individual solutions and their interactions is not necessary, some integral properties of these solutions are essential to single out the stable one. For this purpose, one has to obtain from the mamentum and 
energy equations, the power integrals which are nothing but the entropy and energy balance equations. From this, it is easy to obtain the most general stability criterion far convection which in turn determines the stable solution based on the following physical phenomena:

(i) The stable solution produces more entropy per unit time from the mean temperature field of any other solution than the one produced by its own mean field (Malkus and Veronis 1958; Rohini 1979).

(ii) The stable solution has a greater mean-square gradient than any other solutions (Rudraiah and Srimani 1980). The degeneracy will be removed if there is only one solution.

(iii) For a stable solution, the rate of dissipation of kinetic energy minus a quantity proportional to the rate of increase of entropy by thermal diffusion is maximum (Rudraiah and Srimani 1980; Rohini 1979).

\section{(b) Convection driven by surface tension variations}

A body force is absolt:tely necessary for the buayancy-driven convection discussed abcve. There is, however, another type of convection which does not depend on the presence of a body force (see Scriven and Sternling 1960 for a review). This is interfacial or surface tension-driven motion which obviously demands the presence of a liquid-liquid or liquid-gas interface in addition to a temperaturesensitive interfacial tension. In recent years it was believed that many of the cellular phenomena observed by Benard were probably due not to buayancy force but to variation of surface tension with temperature. This was pointed out by Pearson (1958) and Block (1956), who has developed a linearsied theory, that surface tension forces suffice to cause convection in a liquid layer with a free surface, provided there is a temperature or concentration gradient of the proper sense and sufficient magnitude. Pearson's theary agrees in many essentials with experimental findings. Pearson (1958) have illuminated a neglected type of surface tensiondriven convection. Later Scriven and Sternling (1964) have extended Pearsan's small-disturbance analysis to a still idealised, yet more realistic model of the fluid interface, establishing the effects of finite mean surface tension and surface viscosity. Their analysis is based on a Newtonian fluid interface in which the local departure from equilibrium interfacial stress is directly proportional to the local rate of interfacial strain. By accounting for the possibilities of shape deformations of the free surface, Scriven and Sternling (1964) found that there is no critical Marangoni number for the onset of stationary instability and that the limiting case of zero-wave number is always unstable. They have shown that the effect of surface viscosity of the Newtonian interface is to inhibit stationary instability.

It is of interest to note that in a layer of fluid heated uniformly from below and bounded on both sides by rigid boundaries, convection sets in only due to buoyancy forces. However, if a layer of liquid is bounded in one side by a free surface and on the other by a rigid boundary, convective motion may be induced by a surface tension force due to finite curvature or by the variation of surface tension from point to point.

When a layer of liquid, bounded below by a rigid wall and above by a free surface, is heated uniformly from below, the hot liquid rises to the free surface and cools as it moves along the surface. Thus, the temperature can vary along 
the upper surface. Since the surface tension depends on the temperature (for a liquid it decreases with increasing temperature), there is also a surface tension gradient along the free surface. This induces a surface traction which either tends to pull the fluid along leading to instability or restrain the fluid motion leading to stability. In shallow layers of the fluid, the surface tension irstability can be produced at temperature gradients which are much smaller than those required for buoyancy driven convection. In fact, Koschmieder (1974) studied a shallow layer of silicone-oil on a plane circular copper plate uniformly heated from below and experimentally found that surface-tension forces can produce an array of hexagonal cells of much greater regularity than that observed in buoyancy-driven convection. The fluid moves towards the surface at the centre of each of the hexagons and away from it around their peripheries. Benard's original observations of ordered hexagonal cells are inconsistent with the buoynacy mechanism because of very law temperature gradients, but are consistent with the surface tension mechanism. In the surface tension-driven mechanism, the setting up of convection is expressed by a dimensionless number, $M=\sigma_{s} \Delta T d \mid \rho v \kappa$, called the Marangoni number where $\sigma_{e}$ is the surface tension (force/length) and all other quantities are as defined earlier. The convection sets in at the critical Marangoni number.

In small scale fluid mechaniss, for example, pettoleum engineering, chemical engineering, biology and so on, an understanding of the rolls produced by the combined buoyancy and surface tension forces is essential. The combined effect of these rival theories has been recently investigated by Nield (1964) using linear perturbation technique and he has concluded that, for the case of linear density variation, the coupling between the buoyancy and surface tension effects causing instability reinforce one another and are tightly coupled. Later $\mathrm{Wu}$ and Cheng (1976) extended Nield's linear stability analysis for a horizontal liquid layer considering surface tension and buoyancy effects for the case of water with maximum density effect for the temperature range $0-30^{\circ} \mathrm{C}$. Nield's result shows that the form of the relation between the Marangoni number and the Rayleigh number is rather a weak function of the Biot number $\left(q_{0} d / \kappa, q_{0}\right.$ is the rate of change with temperature of the time rate of heat loss per unit area from the free upper surface). But this is not so in the case of $\mathrm{Wu}$ and Cheng's investigation.

We note that the linear theory discussed by Nield (1964) and $\mathrm{Wu}$ and Cheng (1976) is inadequate to predict the amplitude of such a steady-state solution, because it assumes explicitly that amplitudes are vanishingly small, and hence a nonlinear theory predicting steady amplitudes as a function of Rayleigh and wavenumbers should be cansidered. A general discussion of nonlinear theory with surface tension has not been given much attention and is still an open question.

\section{(c) Convection with internal heat generation}

So far, we have discussed convection due to buoyancy or surface tension or combined buoyancy and surface tension farces in which the fluid temperature decreases linearly with height. In other words, the heat transport in the quiescent state is purely by conduction. $H_{0}$ wever, in many practical situations like the extraction and utilisation of geothermal energy, nuclear reactors, subterranian porous layers and in the applications of material sciences in space, it is of interest to determine 
in what way the instability would be affected if the quiescent state was characterised by a nonlinear temperature profile. Such a nonlinear profile could arise (Sparrow et al 1964; Tritton and Zarraga 1967; Roberts 1967; Nield 1975; Rudraiah et al 1979,1980 ) if there is internal heat generation within the fluid due to heat sources or due to rapid heating, or cooling, at the boundaries. In such situations, instead of heating just from below, we have to consider heat generation throughout the body of the fluid and that the heat leaves the fluid layer through the upper surface, so that the stratification is again unstable. Tritton and Zarraga (1967) investigated experimentally the effect of internal heat generation on convection where the motion is due to instability rather than due to the absence of an equilibrium configuration. Two striking results emerged from their experiments. First, the cell structure was, for moderate Rayleigh number, predominantly hexagonal with motion downwards at the centre of each cell. Secondly. the horizontal scale of the convection pattern grew larger as the Rayleigh number was increased above its critical value. Roberts (1967) answered these experimental challenges using the nonlinear theory and has thrown more light on the advantages and limitations of his appraximate theory of finite-amplitude convection.

A nonlinear temperature distribution also arises due to rapid heating or cooling at a boundary. Theoretical studies of instability with such a nonlinear basic temperature profile were made by Sutton (1950), Marton (1957), Goldstein (1959), Lick (1965), Currie (1967) and Nield (1975). The effect of nonlinear basic tempe rature distribution on surface tension driven convection was analysed by Vida and Acrivos (1968) and by Nield (1975).

Little work has been dane on the combined effect of Coriolis force and nonlinear basic temperature distribution on surface tension driven convection. This is analysed in the present paper. Since the Coriolis force and the non-uniform basic temperature gradient arising from sudden heating or caoling are inherent in all spacecraft environments, the results obtained in this paper will be useful for material science processing in space.

\section{Formulation of the problem}

In this section, we consider the basic equations and the corresponding boundary conditions. For this, we consider an infinite homogeneous liquid layer of uniform thickness $d$ extending to infinity in the $x$-direction and ratating with a constant angular velocity $\vec{\Omega}$ about the $z$-axis which is transverse to the layer. The lower surface $z=0$ is in contact with a fixed rigid plane and the upper surface $z=d$ is free. The only physical quantities that are assumed to vary within the fluid are the temperature, the surface tension, which are regarded as functions of temperature only, and the rate of heat loss from the surface, which is also a function of temperature only. The basic temperature profile is nonlinear due to sudden heating (or cooling) at a boundary.

\subsection{Basic equations}

The basic equations of motion are:

$$
\frac{\partial \vec{q}}{\partial t}+(\vec{q} \cdot \nabla) \vec{q}=-\frac{1}{\rho} \nabla p+v \nabla^{2} \vec{q}-\frac{2}{\rho} \vec{\Omega} \times \vec{q}
$$




$$
\begin{aligned}
& \nabla \cdot \vec{q}=0, \\
& \left.\frac{\partial \tilde{T}}{\partial t}+\overrightarrow{(q} \cdot \nabla\right) \bar{T}=\kappa^{2} \bar{T}
\end{aligned}
$$

where $\vec{q}=(u, v, w)$ is the velacity field,

$p=$ the total pressure

$\tilde{T}=$ the temperature,

$\rho=$ the density of the fluid,

$v=\mu / \rho$ the kincmatic viscosity of the fluid,

$\mu=$ the viscosity of the fluid,

$\kappa=k / \rho$ the thermal diffusivity,

$k=$ the thermal conductivity,

$\vec{\Omega}=(0,0, \Omega)$ is the uniform angular speed of the system,

$\nabla \equiv \frac{\partial}{\partial x} \hat{i}+\frac{\partial}{\partial y} \hat{j}+\frac{\partial}{\partial z} \hat{k}$,

$\nabla^{2} \equiv \frac{\partial^{2}}{\partial x^{2}}+\frac{\partial^{2}}{\partial y^{2}}+\frac{\partial^{2}}{\partial z^{2}}$

$(\hat{i}, \hat{j}, \hat{k})=$ unit vectors in the direction of the space variables $(x, y, z)$.

\subsection{Roundary conditions}

The boundary conditions on velocity are obtained from mass balance, the noslip condition and the stress principle of Cauchy, depending on whether the fluid layer is bounded by either rigid or free surfaces.

2.2a. Rigid surfaces. If the layer is bounded above and below by rigid surfaces, the no-slip condition is valid and we have

$$
u=v=w=0 \text { at } z=0 \text { and } d
$$

where $d$ is the thickness of the layer.

In the study of the onset of convection, it is customary to assume the solution in the form

$$
(u, v, w)=f(z, t) \exp i(l x+m y) .
$$

In that case the equation of continuity (2) using (5) and (6), gives

$$
\frac{\partial w}{\partial z}=0 \text { at } z=0 \text { and } d \text {. }
$$

Thus in the case of rigid boundaries, the boundary conditions on velocity are

$$
w=\frac{\partial w}{\partial z}=0 \text {. }
$$


2.2b. Free surfaces. If the layer is bounded by free surfaces, the boundary conditions on velocity can be obtained, by equalising the change of surface-tension due to the temperature variation across the surface to the shear stress experienced by the liquid at the free surface (Pearson 1958). By balancing the surface-tension gradient with shear stress at the surface, we have

$$
\tau_{\alpha z}=\frac{\partial \sigma_{s}}{\partial x}=\mu \frac{\partial u}{\partial z} \text { and } \tau_{\nu z}=\frac{\partial \sigma_{s}}{\partial y}=\mu \frac{\partial v}{\partial z}
$$

where $\sigma_{s}$ is the surface-tension, $\tau_{x z}$ and $\tau_{y z}$ are the shear stresses.

By proper differentiation and using (2), we get

$$
\frac{\partial^{2} \sigma_{s}}{\partial x^{2}}+\frac{\partial^{2} \sigma_{s}}{\partial y^{2}}=-\mu \frac{\partial^{2} w}{\partial z^{2}}
$$

Following Pearson (1958), we can assume that $\sigma_{s}$ can be expanded as the first order in powers of the temperature variations at the surface, in the form

$$
\sigma_{s}=\sigma_{0}-\sigma_{\mathbf{s}} T
$$

where is $\sigma_{0}$ the unperturbed value of $\sigma_{s}$ and

$$
-\sigma_{T}=\left(\frac{\partial \sigma_{s}}{\partial T}\right)_{T=T_{s}}
$$

For most liquias $\sigma_{T}$ is positive, for as the temperature rises, the difference between the liquid and its vapour phase decreases. Hence, the suitable boundary candition at the free surface in the presence of surface tension is

$$
w=0 \text { and } \mu \frac{\partial^{2} w}{\partial z^{2}}=\sigma_{T}\left(\frac{\partial^{2} T}{\partial x^{2}}+\frac{\partial^{2} T}{\partial y^{2}}\right)
$$

which in the non-dimensional case takes the form

$$
w=0 \text { and } \frac{\partial^{2} w}{\partial z^{2}}=M_{a}\left(\frac{\partial^{2} T}{\partial x^{2}}+\frac{\partial^{2} T}{\partial y^{2}}\right)
$$

where $M_{a}=\frac{\sigma_{T} \triangle T d}{\mu \kappa}$

is the Marangoni number.

In the absence of variation of surface-tension with temperature the boundary conditions on velocity at the frec-surface are

$$
w=\frac{\partial^{2} w}{\partial z^{2}}=0 \text { at } Z=0 \text { and } d
$$

2.2c Thermal boundary conditions. In the study of convection, the thermal conditions applied at the upper and lower surfaces of the fluid are based on the supposition that these surfaces are in contact with the matcrials of infinite thermal conductivity and heat capacity. For, the temperature at the surface is not perturbed when the quiescent state breaks down. A more general thermal houndary condition is

$$
T=\lambda \frac{\partial T}{\partial z}
$$


where $\lambda$ is a constant depending on the thermal properties of the boundary and the liquid. The extreme cases $\lambda=0$ and $\lambda^{-1}=0$ are limiting approximations for temperature perturbations to a very good and bad conductor respectively. In practice, these are referred to as the isothermal and adiabatic cases. In the case of free surfaces, however, the actual physical situation, viz., the heat exchange between the surface and envitonment, su ggests that the standard thermal boundary condition of fixed temperature (i.e., isothermal) may be too restrictive. In that case, adiabatic boundary conditions

$$
\frac{\partial T}{\partial z}=0 \text { at } z=0 \text { and } d
$$

are more realistic.

\subsection{Dimensionless parameters}

Considerable insight into the qualitative nature of the prablem can be obtained from dimensional analysis. The dimensionless parameters determined from the basic equations and boundary conditions help to understand very complex phenomenon. The following non-dimensional parameters are used in the study of convection problems.

(i) The Rayleigh numbər

$$
\mathrm{Ra}=\gamma g \beta d^{4} / v \kappa
$$

where $\beta$ is the adverse temperature gradient maintained between the upper and lower boundaries, $\gamma$ is the volumetric expansion coefficient, $d$ is the depth of the layer, $\kappa$ is the thermal diffusivity and the other quantities are as defined before. Physically, the Rayleigh number represents the balance of energy released by buoyancy force to the energy dissipation by viscous friction and thermal dissipation. The Rayleigh number appears in the energy cquation, so that the heat transfer is related to it.

(ii) The Marangoni number

$$
M_{a}=\frac{\sigma_{T} \triangle \underline{T d}}{\mu \kappa}
$$

where $\sigma_{T}=\partial \sigma / \partial T$ is the rate of decrease of surface tension with increasing temperature, and other quantities are as defined earlier. This Marangoni number is independent of gravity and physically it represents the ratio of surface tension force to dissipative forces.

(iii) The Prandtl number

$$
\sigma=\frac{v(\rho c)}{\kappa}
$$

which is a measure of diffusion of vorticity to heat. In convection problems although the mechanism of the release of thermal energy driving convection is basically simple, a rich variety of phenomena is exhibited by nonlinear convective motions. This variety stems particularly from the dependence of the motion on 
the Prandtl number. At high Prandtl numbers, the nonlinear terms in the equations of motion are of minor importance and the properties of convection are dominated by thermal boundaries. At Prandtl numbers of the order of unity and lower, the momentum terms cause a transition from steady convective rolls to timedependent oscillatory conrection.

(iv) The Grashof number

$$
G_{r}=\frac{\gamma g \beta d^{4}}{v^{2}}=\frac{R_{\mathrm{d}}}{\sigma}
$$

measures the ratio of buayancy force to viscous force. Since the Grashof number appears in the basic cquation of motion, the fluid velocities are directly related to it. For large Grashof numbers $\left(G_{s}>1\right)$, the fluid velocities $v$ can be estimated from

$$
v=\sqrt{\bar{G}_{r}} \cdot \frac{v}{d}=\sqrt{\gamma g \Delta T} d
$$

whereas for small Grashof numbers $\left(G_{r}<1\right)$

$$
v=G_{r} \cdot \frac{v}{d}=\frac{\gamma g \Delta T d^{2}}{v}
$$

(v) The Taylor number

$$
S^{2}=4 \Omega^{2} d^{4} / \nu^{2},
$$

which represents the ratio of coriolis force to viscous force.

(vi) The Nusselt number

$$
\mathrm{Nu}=H / \kappa \beta_{\mathrm{m}}
$$

where $H$ is the vertical heat flux, $\beta_{m}$ is the vertical average of the adverse temperature gradient and $\kappa$ is the thermal diffusivity.

(vii) The Bond number

The relative importance of buoyency or surface tension in setting up of convective motion can be estimated by the dimensionless number

$$
\boldsymbol{B}_{0}=\rho g d^{2} / \sigma_{T}
$$

called the Bond number, which is the measure of the ratio of gravitational to surface tension forces.

\subsection{Simplification of nonlinear forces}

The equations of motion (1) to (4) are highly nonlinear and hence the determination of solution either analytically or numerically is very complicated. To understand the physical insight with reasonable mathematics, usually some assumptions are made. One of the assumptions is that the maximum temperature fluotuations from the mean must be small. In terms of the dimensionless parameters this amounts to saying that the deviation of the critical Marangoni number $M_{a}$ 
from the Marangoni number is small (i.e., $M_{a}-M_{a v}<1$ ). This assumption implies that the nonlinear terms $(\vec{q} \cdot \nabla) \vec{q}$ and $(\vec{q} \cdot \nabla) T$ in equations (1) and (3) can be divided into terms which are finite when averaged over a horizontal plane and into terms of zero average. To achieve this, we let

$$
T^{*}=T(z)+T(x, y, z, t)
$$

If the bar over a quantity denotes the average over a horizontal plane

$$
\left(=\frac{1}{4 \pi l m} \int_{-i}^{l} \int_{-m}^{m}() d x d y\right)
$$

then we have

$$
\bar{T}^{*}=T(z), \overline{T(x, y, z, t)}=0,
$$

substituting (16) into (3) and dividing by $(\rho c)$; we get

$$
\frac{\partial T}{\partial t}-\kappa \nabla^{2} T-\kappa \frac{\partial^{2} T(z)}{\partial z^{2}}=\beta w-(\vec{q} \cdot \nabla) T
$$

where $\quad w=\vec{q} \cdot \hat{k}, \beta=-\frac{\partial T(z)}{\partial z}$

is the negative vertical gradient of mean temperature. Taking the horizontal plane average of $(18)$, we get

$$
-\kappa \frac{\partial^{2} T(z)}{\partial z^{2}}=-\frac{\partial}{\partial z}(\overline{w T(z)})
$$

which on integration yields

$$
\kappa \beta+\overline{W T}=H,
$$

where $H$ is the vertical heat flux in the fluid.

Thus, taking the vertical average

$$
\left(=\frac{1}{d} \int_{0}^{d}\{f\} d z\right)
$$

of (20) we get

$$
\kappa \beta_{m}+(\overline{w T})_{m}=H
$$

where the suffix $m$ denotes the vertical average. From (20) and (21), we get

$$
\frac{\beta}{\beta_{m}}=1+\frac{1}{\kappa \beta_{m}}\left[(\overline{w T})_{m}-(\overline{w T})\right] \text {. }
$$

Equation (18) using (19) and (22) becomes

$$
\frac{\partial T}{\partial t}-\kappa \nabla^{2} T-\beta_{m} w=\frac{\left.(\overline{(w T})_{m}-(\overline{w T})\right)}{\kappa} w-M h
$$


where

$$
h=(\vec{q} \cdot \nabla) T-\frac{\partial}{\partial z}(\bar{w} \bar{T})
$$

is the zero-average beat convection term.

Eliminating pressure $p$ in (1), we get

$$
\frac{\partial}{\partial t}\left(\nabla^{2} w\right)+2 \Omega \frac{\partial \zeta}{\partial z}=v \nabla^{4} w+L
$$

where $\quad L=\frac{\partial}{\partial z}\left[\frac{\partial}{\partial x}(\vec{q} \cdot \nabla) u+\frac{\partial}{\partial y}(\vec{q} \cdot \nabla) v\right]-\nabla_{i}^{2} \vec{q} \cdot \nabla w$,

$$
\nabla_{1}^{2}=\frac{\partial^{2}}{\partial x^{2}}+\frac{\partial^{2}}{\partial y^{2}},
$$

and $\quad \zeta=\frac{\partial v}{\partial x}-\frac{\partial u}{\partial y}$,

is the vertical component of vorticity. From the first two equations of (1), we can get an equation relating $w$ and $\zeta$ in the form

$$
\frac{\partial \zeta}{\partial t}-v \nabla^{2} \zeta-2 \Omega \frac{\partial w}{\partial z}=-Z
$$

where $Z=\frac{\partial}{\partial x}(\vec{q} \cdot \nabla) v-\frac{\partial}{\partial y}(\vec{q} \cdot \nabla) u$

is a zero-average nonlinear term.

The lacal nonlinear stability is usually investigated using the solutions of the form

$$
\begin{aligned}
& w=\epsilon w_{0}+\epsilon^{2} w_{1}+c^{3} w_{2}+\cdots, \\
& v=\epsilon v_{0}+\epsilon^{2} v_{1}+\epsilon^{3} v_{2}+\cdots, \\
& u=c u_{0}+\epsilon^{2} u_{1}+c^{3} u_{2}+\cdots, \\
& T=\epsilon T_{0}+c^{2} T_{1}+c^{3} T_{2}+\cdots, \\
& M_{a}=M_{a 0}+\epsilon M_{a 1}+\epsilon^{2} M_{a 2}+\cdots,
\end{aligned}
$$

where $\epsilon$ is a constant parameter satisfying the suitable boundary conditicns. The first term in each (26) corresponds to the linear stability analysis which is studied in the next section.

\section{Candition for the onset of surface tension driven convection}

The condition for the onset of convection can be determined using the linear stability analysis. This is connected with the solutions of the first-order equations in (26) where the amplitude varies exponentially with time. In other words, for first order solutions to be complete, it is necessary that the parameter $\epsilon$ in (26) must be proportional to the amplitude of the disturbance and this amplitude must 
be infinitesimal. Neglecting the non-linear terms in (23) to (25) and making the equations dimensionless using $d$ as the length scale, $d^{2} / \kappa$ as the time scale, $\kappa / d$ as the velocity scale and $\mu \kappa / \sigma_{\mathbf{r}} d$ as the temperature scale, we get

$$
\begin{aligned}
& \left(\partial / \partial t-\nabla^{2}\right) T-M_{a}^{1 / 2} a f(z) w=0 \\
& \left(1 / \sigma \partial / \partial t-\nabla^{2}\right) \zeta-S \frac{\partial w}{\partial z}=0, \\
& \left(1 / \sigma \partial / \partial t-\nabla^{2}\right) \nabla^{2} w+S^{2} \frac{\partial^{2} w}{\partial z^{2}}=0 .
\end{aligned}
$$

The dimensional temperature gradient $f(z)$ must satisfy

$$
\int_{0}^{1} f(z) d z=1
$$

The scales for $w$ and $T$ have been chosen such that $M_{a}$ appears symmetrically in (27) and in the boundary conditions (see below) rather than in just the cnergy equation or the boundary condition. This choice enables us to establish a variational principle for the present set of equations and as Finlayson (1972) shows, this leads to the conclusion that the eigenvalue $M_{a}$ is stationary in the Galerkin method which we shall apply below.

We now apply the Galerkin method as described by Nield (1975). It is shown that the consideration of even a single term in the expansions of $w$ and $T$ would give an accurate estimate for the critical value of $M_{a}$ in certain cases. In other words, we set $w=A W_{1}$ and $T=B T_{1}$ where $W_{1}$ and $T_{1}$ are suitably chosen trial functions and $A$ and $B$ are arbitrary constants. The presence of rotation, as explained in $\S 1$, sets up overstable motions only for small values of the Prandtl number $\sigma$ (see Veronis 1966; Rudraiah and Rohini 1975). For other values of Prandtl numbers, however, overstable motion is not possible and the principle of exchange of stability is valid, i.e., marginal stability is valid. The present analysis deals with the marginal stability. The marginal stability solution is the one for which the time derivatives in the differential equations (27) to (29) are zero.

Assuming the solutions for $w$ and $T$ in the form

$$
F(z) \exp (i(l x+m y))
$$

equations (27) and (29) take the form

$$
\begin{aligned}
& \left(D^{2}-a^{2}\right)^{3} w+S^{2} D^{2} W=0, \\
& \left(D^{2}-a^{3}\right) T+a M_{a}^{1 / 2} f(z) w=0
\end{aligned}
$$

where $\quad D=\frac{d}{d z}, a^{2}=1^{2}+m^{2}$ and $S^{2}=\frac{4 d^{4} \Omega^{2}}{v^{2}}$ is the Taylor number.

The boundary conditions for a rigid bottom and a free upper surface with temperature-dependent surface tension, each subject to a constant heat flux, are

$$
\begin{aligned}
& W=D W=D T=0 \text { at } z=0 \\
& W=D^{2} W+M_{a}^{1 / 2} a T=D T=0 \text { at } z=1 .
\end{aligned}
$$


Multiplication of (30) by $W$ and (31) by $T$ and integration of the resulting equation by parts with respect to $z$ (from 0 to 1 ) yields, after making use of the boundary conditions, the following:

$$
\begin{aligned}
& 3 a^{3} M_{a}^{1 / 2} D W(1) T(1)=-\left\langle D^{3} W\right\rangle^{2}+3 a^{3}\left(D^{2} W\right)^{2}+\left(3 a^{4}+S^{3}\right)(D W)^{2} \\
& \quad+\left\langle a^{6} w^{2}\right\rangle \\
& M_{a}^{1 / 2} a\langle f(z) W T\rangle=\left\langle(D T)^{2}+a^{2} T^{2}\right\rangle
\end{aligned}
$$

where the angle bracket \langle\rangle denotes the integration with respect to $z$ in the limit 0 to 1 .

Substituting $W=A W_{1}$ and $T=B T_{1}$ into (34) and (35), eliminating $A$ and $B$ and dropping the suffixes, we get

$$
M_{u}=\frac{\left\langle D^{3} W\right\rangle^{2}+3 a^{2}\left(D^{2} W\right)^{2}+\left(3 a^{4} S^{2}\right)(D W)^{2}+\left\langle a^{6} W^{2}\right\rangle\left\langle(D T)^{2}+a^{2} T^{2}\right\rangle}{3 a^{4} D W(1) T(1)\langle f(z) W T\rangle} .
$$

We select the trial functions as $W=(1-z) z^{2}$ and $T=1$ so that they satisfy all the boundary conditions except the one given by $D^{2} W+M_{i}^{12} a T=0$ at $z=1$ and a residual from this equation is included in a residual from the differential equation. The term on the left-hand side of (34) represents this residual.

Substituting these trial functions into (36) we get

$$
M_{u}=\frac{x^{3}+42 x^{2}+1260 x+14 S^{2}+3780}{315 x\left(f(z)\left(z^{2}-z^{3}\right)\right)^{-}},
$$

where $x=a^{2}$.

For any given $f(z), M_{a}$ attains its minimum when $a_{\mathrm{c}}=x_{c}^{2}$, where $x_{\mathrm{c}}$ satisfies the equation

$$
x^{3}+21 x^{2}-\left(7 S^{2}+1890\right)=0 .
$$

\subsection{Onset of convection for various temperature profiles}

\section{Case 1: Uniform temperature gradient}

For uniform temperature gradient, that is for the linear basic temperature profile $f(z)=1$ and (37) takes the form

$$
M_{a}=\frac{12}{315 x}\left[x^{3}+42 x^{2}+1260 x+14 S^{2}+3780\right] .
$$

The critical wavenumber and the corresponding Marangoni number denoted by (Mac) vary with the Taylor number as shown in table 1 of $\S 4$.

\section{Case 2: Piece-wise basic temperature profile for heating from below}

When the layer of liquid is heated from below at a constant rate, we know (Nield, 1975) that the non-u niform basic temperature gradient $f(z)$ is not only non-negative but also decreases monotonically. Thus, we are interested in knowing which 
temperature profile gives the least $M_{a^{0}}$ subject to $f(z) \geqslant 0$. Recently, Nield (1975) has demonstrated that the piece-wise linear profile with $f(z)$ given by

$$
f(z)= \begin{cases}1 / \epsilon & \text { for } 0 \leqslant z<\epsilon \\ 0 & \text { for } \epsilon<z \leqslant 1\end{cases}
$$

is the appropriate one, in the absence of Coriclis force. Even in the presence of Coriolis force, we can demonstrate that this picce-wise lincar profile given by (40), with $\epsilon$ suitably chosen, is the appropriate one, at least for disturbances of small wavenumbers.

Thus, for the bottom heating piece-wise linear profile, substituting (40) into (37), we get

$$
M_{a}=\frac{12\left(x^{3}+42 x^{3}+1260 x+14 S^{3}+3780\right)}{315 x\left(4 \epsilon^{3}-3 c^{3}\right)} .
$$

Then the critical Marangoni number is given by

$$
M_{\mathrm{uc}}=\frac{\triangle}{\max \left(4 \epsilon^{2}-3 \epsilon^{3}\right)}
$$

where $\Delta=\left(M_{a c}\right)_{1}$ for linear profile discussed above. But

$$
\max \left(4 \epsilon^{2}-3 \epsilon^{3}\right)=1.0534977 \text {. }
$$

Thus, as $\epsilon$ increases from 0 to $1, M_{a 0}$ decreascs from $+\infty$ to a minimum value of

$$
\left(M_{\text {ac }}\right)_{2}=\frac{\triangle}{1 \cdot 0534977}
$$

at $\epsilon=0.8889$, and then increases to $\triangle$ at $\epsilon=1$.

Case 3 : Piecewise basic temperature profile for cooling from above

When the layer of liquid is cooled from above at a constant rate, the temperature gradient is not only non-negative but also monotonically dccreasing. In this cesce, the piece-wise linear profile is

$$
f(z)=\left\{\begin{array}{ll}
0 & 0 \leqslant z<1-\epsilon \\
\epsilon^{-1} & 1-\epsilon<z \leqslant 1
\end{array} .\right.
$$

Substituting this in (37), we get

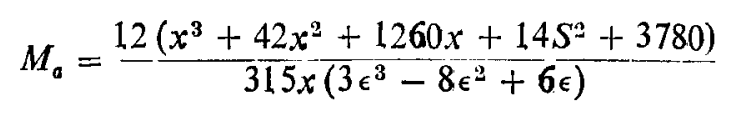

and the corresponding critical Marangoni number is

$$
M_{\text {ab }}=\frac{\Delta}{\operatorname{Max}\left(3 \epsilon^{3}-8 \epsilon^{2}+6 \epsilon\right)} .
$$

As $\epsilon$ increases from 0 to $1, M_{a c}$ decreascs from $+\infty$ to a minimum value of

$$
\left(M_{a c}\right)_{3}=\frac{\triangle}{1.380} \text { at } \epsilon=0.5375
$$


and then increases to $\Delta$ at $\epsilon=1$. Comparing (47) with (43), we find that cooling from above is mare effective, as expected, in reducing eigenvalue, than heating from below.

\section{Case 4: Parabolic basic temperature profile}

In the absence of rotation Debler and Wolf (1970) have considered the problem with a parabalic distribution in which the basic temperature gradicnt is zero at the lower boundary, for which $f(z)=2 z$. Even in the presence of Coriolis force, the parabolic basic temperature distribution leads to $f(z)=2 z$. In this case (37) takes the form

$$
M_{c}=\frac{10\left(x^{3}+42 x^{2}+1260 x+14 S^{2}+3780\right)}{315 x} .
$$

Then the critical Marangoni number is

$$
\left(M_{0.0}\right)_{4}=\frac{\triangle}{1 \cdot 2} .
$$

Comparing (39), (43), (47) and (49) we find that

$$
\left(M_{\theta \theta}\right)_{3}<\left(M_{a \theta}\right)_{4}<\left(M_{a \theta}\right)_{2}<\left(M_{a \theta}\right)_{1} .
$$

Case 5 : Inverted parabolic temperature profile

For inverted parabolic profile $f(z)=2(1-z)$ (see Nield, 1975, pp. 448), (37) takes the form

$$
M_{s}=\frac{15}{315 x}\left(x^{3}+42 x^{2}+1260 x+14 S^{2}+3780\right),
$$

and the corresponding critical Marangoni number is

$$
\left(M_{\mathrm{ac}}\right)_{5}=1 \cdot 25 \triangle \text {. }
$$

Comparing this with the earlier results we find that as expected on physical grounds, inverted parabolic basic temperature profile is more stabilising. Thus, this profile is suitable for suppressing the onset of convection driven by surface tension. This result is of immense utility in material science experimental configurations in space.

\section{Case 6 : Step-function basic temperature profile}

We consider the step-function profile in which the basic temperature drops suddenly by an amount $\Delta T$ at $z=\epsilon$, but is otherwise uniform, and is of the form

$$
f(z)=\delta(z-\epsilon),
$$

where $\epsilon$ is the value of $z$ at which $w T$ has a maximum and $\delta$ denotes the Dirac delta function. In this case (37) takes the form

$$
M_{a}=\frac{x^{3}+42 x^{2}+1260 x+3780+14 S^{2}}{315 x\left(\epsilon^{2}-\epsilon^{3}\right)} .
$$


Then the critical Marangoni number is

$$
M_{a 0}=\frac{\triangle}{12 \operatorname{Max}\left(\epsilon^{2}-\epsilon^{3}\right)}
$$

which has a minimum value

$$
\left(M_{a 0}\right)_{6}=\frac{\Delta}{1.7778} \text { attained a.t } \epsilon=0.6667 .
$$

Thus, the most unstable basic temperature profile, for which $f(z) \geqslant 0$ everywhere, is the step-function profile for which the step occurs at the level at which $w$ is maximum, since $T$ is constant in our appraximation.

\section{Conclusions}

The single-term Galerkin method provides a quick means for obtaining the above results in the presence of Corialis force with different basic temperature profiles. The results (39), (43), (47), (49), (50) and (54) give the critical wavenumbers and the corresponding Marangoni numbers which vary with the Taylor number. These are numerically evaluated for different values of $S^{2}$ and the results are tabulated in table 1.

When the basic temperature gradient is uniform, the condition for the onset of conveotion driven by surface tension in the presence of Coriolis force was investigated by Sarma (1979). He obtained exact analytical solutions which are mathematically cumbersome and the critical Marangoni number for different values of $S$ are obtained from them. The results of table 1 , for $\left(M_{a 0}\right)_{1}$ are compared with those of Sarma (1979, figure 4) and a good agreement is found. Thus, even a single term Galerkin expansion employed here gives accurate results. This table also reveals that

$$
\left(M_{a b}\right)_{6}<\left(M_{\Delta c}\right)_{3}<\left(M_{\Delta 0}\right)_{2}<\left(M_{\Delta c}\right)_{1}<\left(M_{d 0}\right)_{5} .
$$

\begin{tabular}{|c|c|c|c|c|c|c|c|}
\hline$S^{2}$ & $a_{c}$ & $\left(M_{a e}\right)_{1}$ & $\left(M_{\mathrm{ge}}\right)_{2}$ & $\left(M_{00}\right)_{3}$ & $\left(M_{\mathrm{Ec}}\right)_{4}$ & $\left(M_{a o}\right)_{5}$ & $\left(M_{a \theta}\right)$ \\
\hline 0 & 0.0000 & $48 \cdot 00$ & $45 \cdot 56$ & $34 \cdot 79$ & 40.00 & $60 \cdot 00$ & $27 \cdot 00$ \\
\hline $10^{-1}$ & $2 \cdot 8399$ & $81 \cdot 24$ & $77 \cdot 12$ & $58 \cdot 89$ & $67 \cdot 70$ & $101 \cdot 55$ & $45 \cdot 70$ \\
\hline $10^{\circ}$ & $2 \cdot 8420$ & $81 \cdot 30$ & $77 \cdot 17$ & 58.93 & $67 \cdot 75$ & $101 \cdot 63$ & $45 \cdot 73$ \\
\hline $10^{1}$ & $2 \cdot 8625$ & 81.89 & $77 \cdot 13$ & $59 \cdot 36$ & $68 \cdot 24$ & $102 \cdot 37$ & $46 \cdot 06$ \\
\hline $10^{2}$ & 3.0418 & $87 \cdot 39$ & $82 \cdot 95$ & $63 \cdot 35$ & $72 \cdot 83$ & $109 \cdot 24$ & $49 \cdot 16$ \\
\hline $10^{3}$ & 3.9482 & $125 \cdot 65$ & $119 \cdot 27$ & $91 \cdot 08$ & $104 \cdot 71$ & 157.06 & 70.68 \\
\hline $10^{4}$ & 5.9691 & $307 \cdot 10$ & $291 \cdot 50$ & $222 \cdot 397$ & $255 \cdot 91$ & 383.87 & $172 \cdot 74$ \\
\hline $10^{6}$ & $13 \cdot 5853$ & $4531 \cdot 45$ & $4301 \cdot 34$ & $3284 \cdot 58$ & $3776 \cdot 21$ & $5664 \cdot 31$ & 2548.94 \\
\hline
\end{tabular}

Table 1. Values of the Crttical Marangoni and wavenumbers for various values of Taylor number. 
Thus, the most unstable basic temperature profile is the one for which the temperature gradient is a Direct delta function and the most stable basic temperature profile is the one for which the temperature profile is an inverted parabola. In the absence of Corialis force, however, Nield (1975) has calculated the critical Marangani number for the inverted parabolic profile and found that $\left(M_{a 0}\right)_{5}=60$ at the critical wavenumber $a_{s}=0$. Comparing this value with the values of $\left(M_{a s}\right)_{5}$ in table 1, we conclude that inciease of the Taylor number suppresses convection. This conclusion is also true for other temperature gradients. These findings substantiate our objective that rotation and a particular choice of basic temperature gradient suppress the onset of canvection driven by surface tension.

Experimental work to confirm the present results is needed. We suggest that using a solution, such as sugar solution, whose concentration acts as the diffusing quantity, rather than heat, would be convenient to carry out the analysis, since the condition of constant mass flux could then be satisfied without any effort.

\section{Acknowledgements}

This work is sponsorcd by the UGC-DSA programme. I am grateful to Profs. $R$ Narasimha and $S$ Ramaseshan of the Indian Institute of Scicnce, Bangalore, for inviting me to participate and give lectures in the Workshop on Materials and Material Processing in Space. I thank my research student Mr S Balachandra Rao for numerical computation of some of the results.

\section{References}

Bannister T C, Grodzka P G, Spradley L W, Bourgeois S V, Heddon R O and Facemire B R 1973 N.A.S.A. Tech. Memo. X-64772

Block M J 1956 Nature, London 178650

Chandrasekhar S 1961 Hydrodynamic and hydromagnetic stagility (Oxford: Clarendon Press)

Currie I C 1967 J. Fluid Mech. 29337

Debler W R and Wolf L F 1970 Trans. ASME, J. Heat Transfer C92 351

Finlayson B A 1972 The method of weighted residuals and variational principles (Academic Press)

Gordzka P G and Bannister T C 1972 Science 176506

Goldstein A W 1959 N.A.S.A. Tech Rep. R. 4

Joseph D D (1976) Stability of fluid motions I and II. Springer Tracts in Natural Philosophy 27

Koschmieder E L 1974 Adv. in Chem Phys. 26177

Lick W $1965 J$. Fluid Mech. 21265

Malkus W R and Veronis G 1958 J. Fluid Mech. 4225

Morton B R 1957 Quart. J. Mech. Appl. Math. 10433

Nield D A 1964 J. Fluid Mech. 19341

Ostrach S 1979 ESA special publication (114) 41

Palm E 1960 J. Fluid Mech. 8183

Palm E 1960 J. Fluid Mech. 8183

Pearson J R A 1958 J. Fluid Mech. 4489

Polezhaev V I 1979 Proc. 3rd European Symp. on Material Science in Space, E.S.A. Sp. 14225

Roberts P H 1967 J. Fluid Mech. 3033

Rohini G 1979 "Stability of fows through Porous media" Ph.D. Thesis, Bangalore University, India

Rudraiah N 1972 Indian J. Pure Appl. Maths. 3681

Rudraiah N and Prabhamani R P 1973 Trans ASME 40 Series E (4) 879

Rudraiah N and Prabhamani R P 1974a Israel J. Tech. 1289 
Rudraiah N and Prabhamani R P 1974b 5th Int. Heat Transfer Conference, Tokyo, Japan CT 3.1 79

Rudraiah N, Chandrasekhara B C, Veerabhadraiah R and Nagaraj S T 1979 Some flow problems in porous media, PGSAM series 2 Bangalore University

Rudrajah N and Srimani P K 1980 Proc. Roy. Soc. London A 373199

Rudraiah N and Vortmeyer D 1978 Warme-und Stoffubertragung 11241

Rudraiah N, Veerappa B and Balachandra Rao S $1980 \mathrm{~J}$. Heat transfer 102254

Sarma G S R 1979 Proc. 3rd European Symp. o' Material Sciences in Space Grenoble 24-27-ESASP142

Sparrow E M, Goldstein R J and Jonsson V K 1964 J. Fluid Mech. 18513

Scriven L E and Sternling C V 1964 J. Fluid Mech. 19321

Scriven L E and Sternling C V 1960 Nature, London 187186

Segel L A and Stuart J T 1962 J. Fluid Mech. 13289

Sternling C V and Scriven L E 1959 Amer. Inst. Chem. Engg. 6514

Stuart J T 1958 J. Fluid Mech. 41

Stuart J T 1964 J. Fluid Mech. 18 (4) 481

Sutton O G 1950 Proc. Roy. Soc. London A204 297

Tritton D J and Zarraga M N 1967 J. Fluid Mech. 3021

Veronis G $1966 \mathrm{~J}$. Fluid Mech. 31113

Vidal A and Acrivos A 1968 Indust. Engng. Chem. Fund 753

Wu B J and Cheng K C 1976 Int. J. Heat Mass Transfer 19559 